\title{
1 Teasing apart the joint effect of demography and natural selection \\ 2 in the birth of a contact zone
}

3

4 Lili Li ${ }^{1 *}$, Pascal Milesi ${ }^{1^{*}}$, Mathieu Tiret ${ }^{1}$, Jun Chen ${ }^{1,6}$, Janek Sendrowski ${ }^{1}$, John Baison ${ }^{2}$, Zhiqiang

5 Chen $^{2}$, Linghua Zhou², Bo Karlsson ${ }^{3}$, Mats Berlin ${ }^{4}$, Johan Westin ${ }^{5}$, Rosario Garcia-Gil ${ }^{2}$, Harry

$6 \quad \mathrm{Wu}^{2,7}$, Martin Lascoux ${ }^{1 \S}$

Author Contributions: ML and PM designed the study; LL, PM, MT, JC and JS analyzed the data; JB, ZC, LZ, BK, MB, JW, RGG, HW performed experiments or field work and contributed data; LL, PM, MT and ML drafted the manuscript. All authors read and approved the final version of the manuscript.

Competing Interest Statement: None

Classification: Major: Biological Sciences, Minor: Evolution; Plant Biology, Genetics

Keywords: Local adaptation; contact zone; Last Glacial Maximum; natural selection; demography

\section{This PDF file includes:}

Main Text

Figures 1 to 3

Tables 1 to 1 


\section{Abstract}

41 Vast population movements induced by recurrent climatic cycles have shaped the

42 genetic structure of plant species. This is especially true in Scandinavia that was

43 repeatedly glaciated. During glacial periods trees were confined to refugia, south

44 and east of the ice sheet, from which they recolonized Scandinavia as the ice

45 melted away. This multi-pronged recolonization led to large contact zones in most

46 species. We leverage large genomic data from 5000 trees to reconstruct the de-

47 mographic history of Norway spruce (Picea abies) and test for the presence of

48 natural selection during the recolonization process and the establishment of the

49 contact zone. Sweden is today made up of two large genetic clusters, a southern

50 one originating from the Baltics and a Northern one originating from Northern Rus-

51 sia. The contact zone delineating these two clusters closely matches the limit be-

52 tween two major climatic regions. This suggests that natural selection contributed

53 to the establishment and the maintenance of the contact zone. To test this hypoth-

54 esis we first used Approximate Bayesian Computation; an Isolation-with migration

55 model with genome-wide linked selection fits the data better than a purely neutral

56 one. Secondly, we identified loci characterized by both extreme allele frequency

57 differences between geographic regions and association to the variables defining

58 the climatic zones. These loci, many of which are related to phenology, form clus-

59 ters present on all linkage groups. Altogether, the current genetic structure reflects

60 the joint effect of climatic cycles, recolonization and selection on the establishment

61 of strong local adaptation and contact zones.

\section{$62 \quad 247$ words}




\section{Significance Statement}

65 Understanding how past climatic events, human actions and evolutionary forces

66 contributed to the present distribution of genetic diversity is crucial to predict their

67 reaction to the current climate crisis. Vast distribution shifts induced by past envi-

68 ronmental changes, local ecological processes, natural selection and human trans-

69 fers contributed to the current distribution of Norway spruce across Northern Eu-

70 rope. Genome-wide polymorphisms from thousands of individuals show that Scan-

71 dinavia was recolonized after the Last Glacial from both south and north. This two-

72 pronged recolonization established a contact zone between two genetic clusters

73 that matches the limit between two major climate zones. The contact zone is

74 shaped and maintained by natural selection on a large number of loci that form

75 blocks of co-adapted loci spread genome-wide.

\section{0 words}




\section{Main Text}

\section{Introduction}

All natural populations are structured to varying degrees and more than ever population genetic structure matters (1). It matters for a large range of issues: population structure is intrinsically related to speciation and local adaptation; it conditions the response of species to environmental changes (e.g. climate change or the occurrence of new diseases) and it severely limits our ability to associate genetic polymorphism to phenotypic variation or environmental factors using genome-wide approaches. Population structure, and more generally demography, also hampers efforts to detect genomic signatures of natural selection and recent studies on human height have shown that rather fine-scale structure, if not accounted for, can lead to wrong inferences on past selection $(1,2)$. There is therefore a strong incentive to develop methods to capture fine-scaled population genetic structure and thereby strengthens inferences on the relative parts played by past demography and selection in the evolution of species. So, how far have we gone in this respect? Undoubtedly, the availability of genome-wide polymorphisms has vastly enhanced our ability to describe population genetic structure. Yet capturing fine-scale population genetic structure and making sense of it still remains a major challenge. In part, the difficulty arises from the fact that genetic diversity is distributed both discretely and continuously $(3,4)$. This dual nature of population genetic structure reflects the plurality of processes that shape population structure: vast and complex population movements in response to past climatic changes, gene flow among populations, and sometimes even between species, local adaptation and, in many plant and animal species, human-mediated individual transfers.

All the aforementioned factors have come into play in the history of many plant species. Here we shall focus on one of the most common boreal species, Norway spruce (Picea abies (L.) H. Karst)) and mor specifically on Scandinavian populations. Since the seminal study of Lagercrantz and Ryman (5), a large number of studies have outlined the most salient features of the demographic history of Norway spruce (6-16). Basically, as already found by (5), current populations emerged from three main glacial refugia located in the Alps, in the Carpathians 
111 and in the Russian plains. This is, of course, a very rough outline and further stud-

112 ies have added more than one twist to it. In particular, recent studies indicated that

113 these main lineages did not evolve independently but instead created many con-

114 tact zones (8). The nature of these contact zones remains to be elucidated: they

115 could simply be a reflection of past distribution shifts, correspond to ecological

116 zones and be associated to local adaptation, or be the result of both processes. At

117 a larger phylogenetic scale, Siberian spruce (Picea obovata) has a major influence

118 in the northern range of $P$. abies with a large introgression zone starting in the

119 Urals and extending quite far westwards $(8,9,14)$. Introgression from $P$. obovata

120 into $P$. abies is lopsided with a much larger contribution at high latitudes $\left(\sim 65^{\circ} \mathrm{N}\right.$

121 and above) than at intermediate ones $\left(\sim 60^{\circ} \mathrm{N}\right)(8)$. The structure of the vast hybrid

122 zone between the two species could, at least in part, be due to differences in eco-

123 logical requirements between the two parental species (17). Second, in more re-

124 stricted geographical areas, recent introductions have also contributed significantly

125 to the genetic composition of local populations. The Swedish breeding program

126 was established by selecting trees with superior phenotypes (aka "plus trees") in

127 natural stands across the whole country. Targeted genome sequencing of the in-

128 dividuals composing the southern part of the breeding program and of individuals

129 sampled across the natural range of $P$. abies revealed that a large proportion of

130 these "plus trees" were recent introductions originating from most parts of the nat-

131 ural range (8). Third, the pattern of differentiation at genotypic, phenotypic, and

132 environmental variables at the site of origin of the trees were highly correlated in-

133 dicating a strong pattern of local adaptation (18), as often observed in forest trees

134 (19). For example, in both Norway and Siberian spruce, Chen et al. $(7,20)$ detected

135 strong latitudinal clines in growth cessation and were able to associate those to a

136 major candidate gene for photoperiodic response, FTL2, and its pattern of expres-

137 sion. Because these populations are recent these results suggest that local adap-

138 tation can be established very rapidly. These initial studies were based on a hand-

139 ful of candidate genes but more recent studies relying on a much larger number of

140 markers (e.g.,18,21) suggest that quantitative traits and phenology related traits

141 have a polygenic inheritance with loci involved in local adaptation distributed 
142 across the genome. It is, however, still unclear whether adaptive genes are ran-

143 domly distributed or clustered in some specific regions of the genome. The latter

144 would, for instance, be expected when two populations that are under stabilizing

145 selection for different optima are linked by gene flow as is often the case in forest

146 trees $(22,23)$.

147 In the present study we sequenced all individuals from the base population

148 of the Swedish $P$. abies breeding program using exome capture (4769 individuals,

$149>500,000$ SNPs), generating an unprecedented large and dense sampling along a

150 latitudinal gradient ranging from $\sim 55^{\circ} \mathrm{N}$ to $\sim 67^{\circ} \mathrm{N}$. It allowed us to analyze popula-

151 tion genetic structure with a very high resolution. More specifically, we were able

152 to test for pattern of isolation-by-distance, identify barriers to gene flow and test

153 whether those reflect physical or environmental barriers or simply historical contin-

154 gencies. We show that Swedish populations of Norway spruce are divided into two

155 main genetic clusters that closely match the two main climatic regions of the coun-

156 try. Coalescent simulations and Approximate Bayesian Computation allowed the

157 rejection of a purely neutral divergence model between the two main clusters. Fur-

158 thermore, genome scans indicate that clusters of loci distributed across the 12

159 linkage groups correspond to areas of high genetic differentiation and are associ-

160 ated to environmental variables. The current distribution of genetic diversity in Nor-

161 way spruce across Sweden therefore appears to be the result of both demographic

162 processes and local adaptation.

\section{Results}

We first investigated global population structure using the whole dataset 167 which comprises 4607 trees from the base population of the Norway spruce breed168 ing program and 162 trees collected across the natural range of $P$. abies and $P$. 169 obovata (Fig. 1A and Supplementary file 1). Both UMAP (24) and ADMIXTURE 170 (25) retrieved three main domains, Boreal, Carpathians and the Alps, and clusters 171 resulting from admixture between these three domains: Central Europe, Russian172 Baltics and Northern Poland (Fig. 1B, C and S1 to S2 in SI Appendix). Sweden is 173 itself divided into two main genetics clusters, one including southern and central 
174 Sweden (CSE) and the other one the northern part of the country (NFE) (Fig. 1 A-

175 C). Many trees in southern Sweden also correspond to recent introductions. All

176 this is concordant with (8) and (21). Despite their current geographical closeness,

177 the CSE and NFE clusters are divergent and CSE is more closely related to the

178 Russia-Baltics cluster than to NFE $\left(F_{S T}=0.009\right.$ and 0.018, respectively, SI Appen-

179 dix Tab. S1). In addition, the large discrepancy in ancestry components found be-

180 tween two putatively hybrid Russian populations located at the same longitude but

181 at different latitudes support a larger contribution of $P$. obovata to NFE cluster than

182 to CSE (Fig. 1B and C). This general pattern is consistent with a recolonization of

183 the Scandinavian peninsula from refugia with different genetic components and

184 through two different routes, a Northern one and a Southern one. To study more

185 finely the genetic structure of the contact zone and identify the evolutionary forces

186 that shaped it, we focused in the rest of the study on the subset of trees that were

187 native to Sweden and belonged to the CSE $(N=974)$ and the NFE $(N=784)$

188 clusters.

Both UMAP and ADMIXTURE implicitly aim to detect discrete genetic clus-

191 ters. However, Norway spruce tends to be continuously distributed and population

192 structure is the result of both isolation-by-distance and discontinuities. To account

193 for this and identify barriers to gene flow, we first used the software conStruct (3)

194 that considers different levels of population genetic structure: layers correspond to

195 clusters in ADMIXTURE but isolation by distance is considered within layers. Inde-

196 pendently of the number of layers considered, a model including isolation-by-dis-

197 tance within layers predicts the genetic variation pattern better than a non-spatial

198 model (SI Appendix Fig. S3). The lowest cross-validation error (five-fold) was

199 found for three layers (SI Appendix Fig. S3) but, in line with the ADMIXTURE results,

200 two ancestry components explained most of the genetic variation and distin-

201 guished southern trees from northern ones (Fig. 1C). The contact zone between

202 these two main clusters occurred between $60^{\circ} \mathrm{N}$ and $63^{\circ} \mathrm{N}$ (Fig. 2A). Contributions

203 from the southern cluster into the northern one can be detected at latitudes as high

204 as $66^{\circ} \mathrm{N}$ while the northern cluster barely contributed to the populations outside of 
205 the contact zone. Finally, populations from high latitudes (close to $67^{\circ} \mathrm{N}$ ) also pre-

206 sented a specific ancestry component (Fig. 2A). Based on ADMIXTURE results

207 this ancestry component probably represents more recent introgression from $P$.

208 obovata into the northernmost $P$. abies populations (Fig. 1C). To visualize the var-

209 iation in effective migration rate across Sweden and detect barriers to gene flow

210 we then fitted the data to a model of isolation-by-distance and estimated effective

211 migration surfaces (EEMS, (26)). The resulting pattern is complex but regions with

212 low effective migration rate (brown areas), correspond to the contact zone already

213 detected by conStruct and to mountainous regions in the north. North-South barri-

214 ers, as the one along the west coast, are likely artifacts dues to the difficulty of

215 EEMS to account for anisotropy (26) (Fig. 2B). To compare gene flow along lati-

216 tude and longitude we quantified the IBD by regressing a function of pairwise $F_{S T}$,

$217\left(F_{S T} /\left(1-F_{S T}\right)\right)$, over the logarithm of distance between populations. According to

218 (27), the inverse of the slope of the regression provides an indirect estimate of

219 dispersal. First, considering all pairs of populations we detected a pattern of IBD,

220 with an estimated dispersal of $209 \pm 33$ individuals (SI Appendix Fig. S4). But, the

221 IBD was much more pronounced along a latitudinal gradient (228 \pm 33 ), than along

222 a longitudinal gradient $(680 \pm 187)$.

223 To investigate whether ecological barriers to gene flow contributed to the

224 establishment of the contact zone we analyzed environmental variation across

225 Sweden. Three climatic zones were delineated (Fig. 2C, see Online methods $\S$

226 "Abiotic environment characterization and climatic zones definition"): the two main

227 ones separate the northern part from the southern part of the country and the dif-

228 ferentiation is mainly explained by temperature-related variables (annual mean

229 temperature, minimum or average temperature of the coldest months, seasonal-

230 ity). The third climatic zone corresponds to the mountainous area and the west

231 coast and is characterized by higher precipitations than the two other climatic

232 zones (Fig. 2C and SI Appendix S5).

233 The genetic contact zone between the Northern (NFE) and the Southern

234 (CSE) clusters almost perfectly overlap the transition between the Northern and

235 Southern climatic zones (Fig. 2C). Based on ancestry components from 
236 ADMIXTURE $(\mathrm{K}=6)$ we computed a hybridization index, $h_{i}$, which varied from 0 ,

237 (full CSE), to one, (full NFE). While most of the CSE $\left(h_{i} \leq 0.33\right)$ or NFE $\left(h_{i} \geq 0.66\right)$

238 trees were restricted to the Southern or the Northern climatic zone, respectively

239 (Wilcoxon's rank-sum test, $\left.W>7 \times 10^{-5} ; p<0.001\right)$, the hybrids $\left(0.33<h_{i}<0.66\right)$

240 were located on the transition zone and evenly distributed between the two climatic

241 zones $(W=789 ; p=0.18$, Fig. $2 C)$. Such a match between the main environmental

242 zones and the genetic structure strongly suggests that natural selection contrib-

243 uted to the creation and maintenance of the contact zone between the two genetics

244 clusters.

245 To test whether natural selection contributed to the establishment and 246 maintenance of the contact zone we simulated different coalescent isolation with

247 migration scenarios and calculated their posterior probabilities with an Approxi248 mate Bayesian Computation ( $A B C$ ) approach implemented in the program DILS 249 (28). Briefly, in the presence of linked selection one expects a larger variance in 250 effective population size, $\mathrm{N}_{\mathrm{e}}$, among loci than under a strictly isolation with migra-

251 tion model. In order to measure the effect of hybridization on demographic scenario 252 inferences, we created three samples of 20 individuals (10 from NFE and 10 from 253 CSE) varying in their distance to the contact zone (far, intermediate, or close). In 254 all three cases the most likely model was one with linked selection, with posterior 255 probabilities of $71.24 \%, 93.39 \%$, and $87.94 \%$ for far, intermediate and close, re256 spectively (Tab. 1). This suggests that linked selection occurred over the entire 257 range of each climate zone. This result was further confirmed by additional forward 258 simulations (SI Appendix Section 2).

259 To identify genomic signatures of local adaptation associated with the 260 contact zone, we then (i) scanned our genomic data for loci with extreme allele 261 frequency differences between geographic regions using Bayenv2 ( $X^{T} X$ score) 262 (29) and pcAdapt (30) and (ii) ran genotype-environment associations (GEA) using 263 Bayenv2 and Ifmm2 (31) on a subset of 142,765 SNPs with MAF > 0.05. Bayenv2 264 is population based while pcAdapt and Ifmm2 are individual-based. Genome scans 265 identified 440 and 990 SNPs showing extreme allele frequency differences 266 between geographic regions, using pcAdapt or $X^{T} X$ statistic, respectively (32\% 
267 overlap at the gene level). With GEA, a total of 1616 (bayenv2) and 1298 (flmm2)

268 SNPs were associated to at least one of the 24 bioclimatic variables (21\% overlap

269 at the gene level). The number of significant associations per bioclimatic variable

270 was correlated between the two analyses (Spearman's rho $=0.53, \mathrm{~S}=1070, p<$

271 0.01) (SI Appendix Tab. S2). Most of the significant associations were with the

272 climatic variables that contributed the most to the discrimination of the two main

273 climatic zones (Spearman's rho $=0.76, S=229.8, p<0.001$ and rho $=0.65, S=$

$274350.15, p<0.01$, respectively for Ifmm2 and bayenv2).

275 The genes putatively involved in local adaptation were tested for gene on-

276 tology term enrichment. They were first grouped into four main categories depend-

277 ing on whether they were (i) differentiation outliers or (ii) associated to tempera-

278 ture-related, (iii) precipitation-related or (iv) seasonality-related climate variables.

279 Enrichment was significant for gene ontology terms associated to biological pro-

280 cesses related to environmental stimulus detection, metabolic pathways, growth

281 and morphogenesis regulation, as well as biotic interactions (SI Appendix, Fig. S6).

282 Since GO term annotation for the $P$. abies genome is incomplete we also adopted

283 an ad hoc approach, specifically focusing on functions of interest, namely, re-

284 sponse to photoperiod, cold or abiotic stimuli, growth, flowering and circadian

285 clock. In total we identified 134 candidates SNPs located within or in the vicinity of

28681 unique genes involved in these functions. We used a heatmap to illustrate how

287 allele frequencies at these SNPs changed across populations. Populations clus-

288 tered according to latitude (SI Appendix, Fig. S7) and this clustering was mostly

289 driven by genes associated to the circadian clock and therefore to phenology and

290 growth rhythm: XAP5 time keeper (Spearman's rho =0.70), flowering-time-like loci

$291(F T L$, rho $=0.59)$, early flowering loci $3(E F L 3$, rho $=0.92)$, early flowering loci 3

292 high $(E F L 3-h i g h$, rho $=0.91)$, sensitivity to red light reduced $1(S S R 1$, rho $=0.78)$

293 and gigantea $($ rho $=0.76)$, (Fig 3A).

In spite of the high fragmentation of the Norway spruce reference genome

296 (32), we successfully mapped 89,940 SNPs onto the Norway spruce genetic map

297 (33). Genes putatively involved in local adaptation clustered in a limited number of 
298 genomic regions spread across the genome (four genes on average per regions,

299 maximum 14 for bayenv2 analysis and six on average and maximum 22 for lfmm2),

300 with one or several clusters on most linkage groups (Fig. 3B and C, SI Appendix,

301 Section 3). All candidate regions with extreme allele frequency differences be-

302 tween geographic regions were associated to at least two environmental variables,

303 suggesting a direct or indirect causal relationship between high genetic differenti-

304 ation and environmental factors. Regions enriched for candidate genes were more

305 often associated to temperature-related variables (on average $4.5 \pm 3.6$ regions

306 across the two GEA analyses, the maximum being nine for temperature annual

307 range) than to precipitation-related ones $(0.94 \pm 1.1$, maximum being three for pre-

308 cipitation of driest quarter). The climatic variables that contributed the most to the

309 discrimination of the two main climatic zones were also those for which we de-

310 tected the highest number of genomic regions enriched for candidate genes

311 (Spearman's $r h o=0.65 ; S=469$.32; $d f=18 ; p=0.002$ for bayenv2). Similar results

312 were obtained with Ifmm2, the number of candidate genomic regions per variable

313 being highly correlated between the two analyses ( $r h o=0.63, S=853, p<0.001$

314 and Figure 3C). Genomic regions associated to local adaptation were found across

315 all linkage groups but formed large clusters on individual chromosomes. Taken

316 together, these results and those of the $A B C$ analysis strongly support a significant

317 contribution of natural selection to the establishment and maintenance of the con-

318 tact zone.

\section{Discussion}

321

322 Contact zones are a rich source of information on the interplay between demogra-

323 phy and selection in shaping the genetic structure of species (34). Leveraging ge-

324 nomic data from almost 5000 trees sampled across Sweden and the natural range

325 of Norway spruce, we reconstructed the origin of the contact zone separating the

326 south and the north of Scandinavia and showed that natural selection acting on

327 gene clusters dispersed across the whole genome contributed to the differentiation

328 between the two main genetic clusters. Given that Norway spruce has been pre-

329 sent in Scandinavia a rather limited number of generations (35), this is an important 
330 result with respect to climate change since, unless trees were pre-adapted before

331 invading Scandinavia, it suggests rapid local adaptation.

\section{A recent contact zone}

The general clustering is congruent with what was observed in earlier stud-

336 ies using smaller sample sizes (8) and different markers (14). According to these

337 population genetics studies and the paleo-ecological record (pollen fossil data but

338 also macrofossils) (35-39), current European populations of $P$. abies originate from

339 at least three main ancient refugia located in the Alps, in the Carpathians and in

340 the Russian and Western Siberia Plains. Introgression from Siberian spruce $(P$.

341 obovata) also contributed significantly to the latter, especially at high latitudes (14).

342 What our data show is that these three lineages did not evolve independently but

343 rather entered into contact at many points. For example, as apparent from the Ad-

344 mixture analysis, both Northern Poland and the Russian-Baltic domain, are three

345 ways admixture, with a major contribution from the Carpathians and more limited

346 contributions from the Alps and P. obovata.

347 The recolonization of Northern Europe by $P$. abies started relatively late and

348 spruce migration rates for Fennoscandia varied between 200 and 500 m.year $^{-1}$

349 (38). Our data supports the existence of two routes of recolonization of Scandina-

350 via, both from east to west, but one entering Scandinavia from the north and mov-

351 ing southward and one entering Scandinavia at a lower latitude and moving both

352 northward and southward (35). The two routes joined between $60^{\circ} \mathrm{N}$ and $63^{\circ} \mathrm{N}$ and

353 created an admixture zone that was identified in the present study. Fossil data

354 indicate that trees entered Scandinavia around 13,000-12,000 years ago from the

355 South and 4,000-3,000 years ago from the North (39). The recolonization of Scan-

356 dinavia by Norway spruce occurred in two phases: a first phase during which small

357 outposts were established and, later on, a second phase when dispersal from

358 those and from a larger front started (39). If their average migration rate was 300

$359 \mathrm{~m} /$ year, trees should have reached the current location of the contact zone after

360 around 3300 years and 2000 years, respectively. So, the contact zone would have 
361 been created some 2000 years ago, or, assuming a generation time of around 50 362 years, some 40 generations ago. The pollen fossil record suggests a somewhat 363 lower migration rate and the fronts reaching central Sweden some 3000 years ago, 364 so around 60 generations ago. Of course, these are approximate dates and we do 365 not expect the northwards and southwards migrations to progress at similar speed 366 since it is a well-established fact that Norway spruce can easily be transferred 367 some 3-4 degrees of latitude north without much loss in growth but that a south368 wards move is generally much less successful (40). We indeed observed an asym369 metry, with the southern cluster contributing to the northern one as high as latitudes $37066^{\circ} \mathrm{N}$ while the northern cluster contribution to the southern one was much more 371 limited. In any case, given that gene flow is important in Norway spruce, this implies 372 that one would likely have expected the contact zone to have started to be eroded 373 by gene flow unless it were maintained by selection.

374 These two recolonization routes are not unique to spruce and are observed 375 in others species, for example, humans, where they are well established $(41,42)$.

376 The resulting admixture zone coincides with a postulated zone of postglacial con377 tact for many plant and animal species $(12,43)$. A similar contact zone is for in378 stance observed in Populus tremula L. (44), brown bears (45) or rodents (46). In

379 all these organisms, the contact zone has been initially interpreted as the meeting 380 point between the two main lineages that recolonized Scandinavia after the Last 381 Glacial Maximum (about 25 Kya). In Populus tremula L., though, the contact zone 382 corresponds to a sharp change in allele frequency at the FTL gene that is involved 383 in the control of budset (47).

In addition to the main contact zone, in the conStruct analysis, populations 386 located north of $62^{\circ} \mathrm{N}$ contain an ancestral component that was specific to those 387 populations (Fig. 2A, red component). A similar result was obtained by (48) who 388 analyzed the genetic diversity at 15 SSR loci in nine of the breeding populations 389 from northern Sweden. The two northernmost of these nine populations formed a 390 separate cluster in a PCA and both populations presented signs of bottlenecks 391 (48). Those populations are characterized by a higher contribution from $P$. 
392 obovata. (11) also observed that trees collected from Northern Fennoscandia and

393 Russia-Urals clustered in a Neighbor-joining tree based on seven SSR loci. Thus,

394 this genetic group reflects how far west $P$. obovata genetic influence was felt, an

395 influence that might have been reinforced locally by bottlenecks during the recolo-

396 nization process (14). This westward recolonization pattern at high latitudes is not

397 specific to the $P$. abies - $P$. obovata species pair. A similar situation is observed

398 between Larix sibirica and Larix gmelinii with introgression of mtDNA from the local

399 species in the west, L. sibirica, into the invading species from the East, L. gmelinii

400 (49-51). This trend does not preclude migration in the opposite direction. For ex-

401 ample, Pinus sylvestris apparently dispersed primary from western Europe (52).

$402 \quad$ Finally, pollen analysis and simulations supported a moving front recoloni-

403 zation of Scandinavia rather than population expansion from local refugia

$404(35,38,53)$. Putative local refugia have been found in mountainous area of central

405 Sweden (54) and might have had a local impact but the fit to an isolation by dis-

406 tance pattern, together with the importance of the contribution of $P$. obovata, would

407 rather argue for a recolonization from populations located outside of the main gla-

408 ciated areas. Also, these refugial populations are made of small trees that repro-

409 duce mainly asexually (54) and it is highly doubtful that they could have contributed

410 massively to surrounding populations. More generally, comparison between Picea

411 and Larix in Eastern Siberia suggests that Picea biology (relatively heavy seeds,

412 low genetic diversity in survival pockets) might explain why Larix and not Picea

413 was capable of population expansion from small, scattered refugia (55).

415 Polygenic architecture of local adaptation along the contact zone

We have so far discussed the data in term of demographic events. However, 418 the major contact zone that was observed in Scandinavia (i) corresponds to a dis419 continuity in bioclimatic factors, (ii) is better explained by a model incorporating 420 linked selection than by a purely neutral one and (iii) is accompanied by a large 421 number of genomic areas containing clusters of genes characterized by high ge422 netic differentiation and association with climatic variables changing across latitude 
423 (e.g. photoperiod, temperature-related climatic variables). While this is not the first

424 study indicating the presence of selection and adaptive cline in forest trees along

425 a latitudinal gradient this is the first one that demonstrates the genome wide impact

426 of local adaptation. The observed pattern is expected under polygenic adaptation

427 for different optima when populations are linked by gene flow $(22,23)$ and could be

428 further reinforced or even caused by structural rearrangements that allow the

429 spread of co-adapted alleles. Unfortunately, the current state of the genome as-

430 sembly (> 1.5 M scaffolds) does not allow us to investigate further this hypothesis.

431 However, as the largest region includes up to 22 genes carried by different scaf-

432 folds, we can expect that some regions enriched for candidate genes are structural

433 variants that can further limit gene-flow between the northern and southern clus-

434 ters.

A large number of genes were significantly associated to environmental variables and differentiation outliers; 205 unique genes carried at least one significant

438 SNPs associated to environmental variables and 91 were outliers in genome 439 scans. In line with (18) and (21), this suggests a high degree of polygenicity of local 440 adaptation in Norway spruce. Because of the confounded effects of population 441 structure and of the main environmental gradient, these numbers are likely under442 estimates (18). The more pronounced pattern of isolation by distance at these loci 443 than at all loci considered jointly strongly suggests that they contribute to local 444 adaptation. This is further supported by the involvement of many of the identified 445 candidate genes in the control of the circadian clock (XAP5, FTL, EFL-3, EFL-3 446 high, Gigantea, CEN1, SRR1, LHY) and therefore in phenology and growth 447 rhythm. Interestingly three important genes for phenology, FTL, EFL-3 and Gigan448 tea, are located close by on linkage group 8 (Fig. 3C). This co-localization could 449 have been favored by the strong selection pressure on juvenile trees exerted by 450 frost in late-spring and early-fall (56). In any case, selection on phenology will in451 duce differences in reproductive period that could partly explain the maintenance 452 of the contact zone by limiting the gene-flow between the two clusters. 
Considering, the overall low population genetic differentiation together with

455 the relatively short time spent by trees in Scandinavia the establishment of such a

456 strong clinal gradient would seem to imply a rather strong selection pressure, even

457 at individual loci. Assuming that i) local refugia did not contribute significantly to

458 the recolonization of Scandinavia, ii) Norway spruce entered Scandinavia around

$45910,000-12,000$ cal. BP and reached central Sweden around 3,000 cal. BP $(11,39)$

460 and iii) considering a generation time of about 50 years implies that the observed

461 gradient at adaptive loci over Sweden was established in around 150-200 genera-

462 tions. However, it cannot be ruled out that pre-adapted loci also contributed to local

463 adaptation in newly-colonized areas. As trees from the two main clusters originate

464 from similar latitudes than the ones found today in Scandinavia, a certain level of

465 pre-adaptation seems likely. Additional samples from northwestern Russia and

466 from the Baltics would be necessary to test this hypothesis.

\section{Practical implications}

The genetic structure of the breeding population is important for the management of its genetic resources, for genome-wide association studies and when

472 establishing training sets for genomic selection (57). All our analyses indicate that

473 the individuals used to establish the current breeding population belong to at least

474 seven main genetic clusters. Southern Sweden is particularly complex due to the

475 presence of a large fraction of recent introductions (8) but central Sweden is more 476 homogeneous (CSE) and the northern part of the country consists of two clusters.

477 Our data therefore suggest that at least three training sets may be sufficient to 478 account for most population genetic structure. Importantly, our results also indicate 479 that the current contact zone is maintained by natural selection and will therefore 480 change as the climate does. Three main scenarios for the reaction of Scandinavian 481 population under rapid climate change seem plausible. First, trees from the north482 ern cluster (NFE) are progressively going to be introgressed with genes from the 483 southern cluster as the latter move northwards and the contact zone will progres484 sively disappear. Second, barriers to gene flow are strong enough between the 
485 two clusters for the contact zone to persist and shift northwards. Third, assuming

486 that growth traits are a good proxy for fitness, global change will advantage popu-

487 lations with more southern origins, for instance favoring trees with an Alpine or

488 Carpathian genetic background and those will progressively replace existing pop-

489 ulations. Given that (18) showed that, at least in the southern and central parts of

490 Sweden, trees with an Alpine or a Carpathian origin outperformed the trees from

491 local provenance for growth traits, this may well occur. This evolution of the contact

492 zone will need to be monitored and incorporated into future genotype-by-climate

493 zone interaction studies for optimizing the delineation of breeding zones, some-

494 thing that, to the best of our knowledge, has not yet been implemented in forest

495 tree breeding.

At any rate, predicting the future evolution of natural populations, for instance for conservation and breeding, is and will remain a complex task, even more

500 The detection of adaptive loci that are associated with phenotypic traits and/or en-

501 vironment will not be sufficient to predict future adaptation under climate change

502 scenarios without a deep knowledge of both global and local genetic diversity and

503 how this diversity translates into fitness under various environments. First, intro-

504 gression from closely related species (or from individuals from outside of the focal

505 range) plays a role in shaping genetic diversity and response to environment. Sec-

506 ond, adaptation to a highly dimensional environment requires a high degree of

507 polygenicity. It is therefore intrinsically challenging to extrapolate both genotype-

508 phenotype and genotype-environment relationships under various scenarios in-

509 volving either demographic or environmental changes. This would require exten-

510 sive studies, at both local and global geographical scales, repeated over time and

511 with an exhaustive sampling of genetic diversity in the target species but also in

512 species with whom it can hybridize. 


\section{Materials and Methods}

518 Sample collection: The study was based on 4769 spruce trees, originating mainly

519 from the Swedish Norway spruce ( $P$. abies) breeding (Figure 1). Among them, 162

520 individuals were collected in natural populations across the Norway spruce natural

521 distribution range (8). The remaining trees (4607) were "plus" trees (trees of out-

522 standing phenotype) sampled in Skogforsk (The Forestry Research Institute of

523 Sweden) plantations across Sweden. These trees were genotyped ((8), BioProject

524 PRJNA511374 and (21), BioProject PRJNA731384) using an exome capture tar-

525 get re-sequencing strategy (40,018 diploid 120bp-length probes designed to cap-

526 ture 26,219 P. abies genes, (58)).

527 Single Nucleotide Polymorphism calling: Raw reads were mapped to the $P$.

528 abies genome reference v1.0 (31) and single nucleotide polymorphisms (SNPs)

529 were identified using HaplotypeCaller v.3.6 (59) and quality filtered. Individuals

530 with more than $50 \%$ missing data were also removed $(\mathrm{N}=282)$. The filtered da-

531 taset included 4508 individuals and 504,110 SNPs. Those SNPs were annotated

532 based on the most recent genome annotation available for $P$. abies (v1.0,

533 http://congenie.org/).

534 Population structure and genotype assignment: For population structure anal-

535 yses, sites in high linkage disequilibrium $\left(r^{2}>0.2\right)$ as well as singletons were re-

536 moved using PLINK v.1.9 (60). Among the remaining SNPs, 155,211 putatively

537 neutral SNPs (i.e. within introns and intergenic regions) were kept for demographic

538 analyses. Population structure was first characterized using a principal component

539 analysis (EIGENSOFT, v.7.2.0 with default parameters,

540 https://github.com/DreichLab/EIG, 61). Trees with unknown geographical origin

541 assigned to a genetic cluster using Random Forest classification as in (8). We also

542 analyzed population structure with ADMIXTURE v1.3 (25) and calculated pairwise

543 fixation indices (Hudson's estimator of $F_{\mathrm{ST}}, 62$ ) between $P$. obovata, admixed $P$.

544 abies $\times$ P. obovata populations and the $P$. abies genetic clusters defined through

545 the UMAP analysis.

546 Spatialized analyses of genetic variation: For the following analyses, only trees

547 that were of confirmed Swedish origin (base on genetic clustering) and with known 
548 geographic coordinates were considered $(\mathrm{N}=1758)$. To consider both discrete

549 clusters and continuous distribution of the genetic variation of Norway spruce

550 across Sweden, we first used the conStruct software v. 1.03 (3) that combines

551 model-based clustering algorithms with an isolation by distance model. To identify

552 corridors or barriers to gene flow, we used EEMS software (v. 0.0.9000, 26) and

553 we quantified the pattern of isolation by distance by regressing a function of $F_{\mathrm{ST}}$

554 (27) over the logarithm of the distance between pairs of populations.

555 The contribution of linked selection to the contact zone: In order to test

556 whether linked selection contributed to the establishment and maintenance of the

557 contact zone, we used the program DILS (28). Briefly, DILS implements an Ap-

558 proximate Bayesian Analysis to compare two-population demographic models and

559 identifies the most likely demographic scenario with and without linked selection.

560 Considering that distance to the contact zone might influence demographic infer-

561 ences (e.g., hybrids have different history than pure individuals), we created three

562 different datasets as inputs for DILS depending on the distance to the contact zone.

563 Testing for local adaptation: First, to assess whether the contact zone between

564 the main genetic clusters corresponded to a shift in abiotic conditions across Swe-

565 den, we defined climatic zones based on 19 bioclimatic records (Chelsa database

566 v1.2, http://chelsa-climate.org/, 30 arc-second resolution). Different approaches

567 were used to test for the presence of local adaptation at the genomic level and to

568 detect association between genomic polymorphisms and environmental variables.

569 To detect genetic differentiation outliers we used the Bayenv2 software $(29,63)$

570 and "pcadapt" v4.3.2 R package $(30,64)$. To detect Genotype-environment asso-

571 ciations we used "Bayenv2" and "Ifmm2" (31). The same 19 Chelsa bioclimatic

572 variables as those used to define the climatic zone as well as derived combination

573 of those were used for each tree location.

574 Candidate genes putative functions and genetic mapping: Gene ontology

575 (GO) enrichment was performed using the 'topGO' R package (v2.44.0; (65)).

576 About $60 \%$ of all SNPs were successfully positioned onto the $P$. abies consensus

577 genetic map (32). We developed a new approach to identify regions enriched for

578 outliers (either low p-values in pcAdapt, and Ifmm2 analyses or high Bayes factor 
579 for Bayenv2). The method (66) is described in online methods and freely accessi-

580 ble at https://github.com/milesilab/peakdetection.

581

582

583

584

585

586

587

588

589

590

591

592

593

594

595

596

597

598

599

600

601

602

603

604

605

606

607

608

609

610

611

612

613

614

615

616

617

618

619

620

621

622

623

624

625

\section{Acknowledgments}

We are grateful to Luis Leal and Linus Söderquist for comments on early versions of the manuscript and to Camille Roux for help with running DILS. This project is supported by the Swedish Foundation for Strategic Research (SSF), grant number RBP14-0040. MT was supported by the EU H2020 project, B4EST. The computation and data handling were provided by the Swedish National Infrastructure for Computing (SNIC) at Uppmax, partially funded by the Swedish Research Council through grant agreement no. 2018-05973.

\section{References}

1. N. Barton, J. Hermisson, M. Nordborg, Population genetics: Why structure matters. Elife 8, e45380 (2019).

2. D. Lawson et al., Is population structure in the genetic Biobank era irrelevant, a challenge, or an opportunity? Human Genetics 139(1), 23-41 (2020).

3. G.S. Bradburd, G.M. Coop, P.L. Ralph, Inferring continuous and discrete population genetic structure across space. Genetics 210(1), 33-52 (2018).

4. A. Diaz-Papkovich, L. Anderson-Trocmé, C. Ben-Eghan, S. Gravel, UMAP reveals cryptic population structure and phenotype heterogeneity in large genomic cohorts. PLoS Genetics 15(11), e1008432 (2019).

5. U. Lagercrantz, N. Ryman, Genetic structure of Norway spruce (Picea abies): Concordance of morphological and allozymic variation. Evolution 44(1), 38-53 (1990)

6. G. Bucci, G.G. Vendramin, Delineation of genetic zones in the European Norway spruce natural range: preliminary evidence. Molecular Ecology 9(7), 923-34 (2000).

7. J. Chen et al., Disentangling the roles of history and local selection in shaping clinal variation of allele frequencies and gene expression in Norway spruce (Picea abies). Genetics 191(3), 865-81 (2012).

8. J. Chen et al., Genomic data provide new insights on the demographic history and the extent of recent material transfers in Norway spruce. Evolutionary Applications 12(8), 1539-51 (2019).

9. Z. Fagernäs, Biogeography of Norway spruce (Picea abies (L.) Karst.): Insights from a genome-wide Study. MSc Thesis Umeå University (2017). 
10. M. Heuertz, et al., Multilocus patterns of nucleotide diversity, linkage disequilibrium and demographic history of Norway spruce [Picea abies (L.) Karst]. Genetics 174(4), 20952105 (2006).

11. M.M. Tollefsrud et al., Combined analysis of nuclear and mitochondrial markers provide new insight into the genetic structure of North European Picea abies. Heredity 102(6), 549-62 (2009).

12. M.M. Tollefsrud et al., Genetic Consequences of Glacial Survival and Postglacial Colonization in Norway Spruce: Combined Analysis of Mitochondrial DNA and Fossil Pollen. Molecular Ecology 17(18), 4134-50 (2008).

13. M.M. Tollefsrud, M. Latałowa, W.O. van der Knaap, C. Brochmann, C. Sperisen, Late Quaternary history of North Eurasian Norway spruce (Picea abies) and Siberian spruce (Picea obovata) Inferred from macrofossils, pollen and cytoplasmic DNA. Journal of Biogeography 42(8), 1431-42 (2015).

14. Y. Tsuda et al., The extent and meaning of hybridization and introgression between Siberian spruce (Picea obovata) and Norway spruce (Picea abies): Cryptic refugia as stepping stones to the West? Molecular Ecology 25(12), 2773-89 (2016).

15. G.G. Vendramin et al., Chloroplast microsatellite analysis reveals the presence of population subdivision in Norway spruce (Picea abies K.). Genome 43(1), 68-78 (2000).

16. X. Wang, C. Bernhardsson, P.K. Ingvarsson, Demography and natural selection have shaped genetic variation in the widely distributed conifer Norway spruce (Picea abies). Genome Biology and Evolution 12(2), 3803-17 (2020).

17. E. Nakvasina, N. Demina, N. Prozherina, N. Demidova, Assessment of phenotypic plasticity of spruce species Picea abies (L.) Karst. and $P$. obovata (Ledeb.) on provenances tests in European North of Russia. Central European Forestry Journal 65(2), 121-28 (2019).

18. P. Milesi et al., Assessing the potential for assisted gene flow using past introduction of Norway spruce in Southern Sweden: Local adaptation and genetic basis of quantitative traits in trees. Evolutionary Applications 12(10), 1946-59 (2019).

19. O. Savolainen, T. Pyhäjärvi, T. Knürr, Gene flow and local adaptation in trees. Annual Review of Ecology, Evolution, and Systematics 38, 595-619 (2007).

20. J. Chen et al., Clinal Variation at Phenology-related genes in spruce: Parallel evolution in FTL2 and Gigantea? Genetics 197(3), 1025-38 (2014).

21. Z.-Q. Chen et al., Leveraging breeding programs and genomic data in Norway spruce (Picea Abies L. Karst) for GWAS analysis. Genome Biology 22(1), 1-30 (2021).

22. S. Yeaman, M.C. Whitlock, The genetic architecture of adaptation under migration-selection balance. Evolution 65(7), 1897-1911 (2011).

23. S. Yeaman, Genomic rearrangements and the evolution of clusters of locally adaptive loci. Proceedings of the National Academy of Sciences 110(19), E1743-51 (2013). 
24. A. Diaz-Papkovich, L. Anderson-Trocmé, C. Ben-Eghan, S. Gravel, UMAP reveals cryptic population structure and phenotype heterogeneity in large genomic cohorts. Plos Genet 15, e1008432 (2019).

25. D.H. Alexander, J. Novembre, K. Lange. Fast model-based estimation of ancestry in unrelated individuals. Genome Research 19(9), 1655-64 (2009).

26. D. Petkova, J. Novembre, M. Stephens, Visualizing spatial population structure with Estimated Effective Migration Surfaces. Nature Genetics 48(1), 94-100 (2015).

27. F. Rousset, Genetic differentiation and estimation of gene flow from F-statistics under isolation by distance. Genetics 145(4), 1219-28 (1997).

28. C. Fraïsse et al., DILS: Demographic inferences with linked selection by using ABC. Molecular Ecology Resources https://doi.org/10.1111/1755-0998.13323 (2021).

29. T. Günther, G. Coop. Robust identification of local adaptation from allele frequencies. Genetics 196(1), 205-20 (2013).

30. K. Luu, E. Bazin, M.G. Blum, Pcadapt: An R Package to perform genome scans for selection based on Principal Component Analysis. Molecular Ecology Resources 17(1), 67-77 (2017).

31. K. Caye, B. Jumentier, J. Lepeule, O. François, LFMM 2: Fast and accurate inference of gene-environment associations in genome-wide studies. Molecular Biology and Evolution 36(4), 852-60 (2019).

32. B. Nystedt et al., The Norway spruce genome sequence and conifer genome evolution. Nature 497(7451), 579-84 (2013).

33. C. Bernhardsson et al., An ultra-dense haploid genetic map for evaluating the highly fragmented genome assembly of Norway spruce (Picea Abies). G3 Genes|Genomes|Genetics 9(5), 1623-32 (2019).

34. K. Johannesson, A. L. Moan, S. Perini, C. André, A Darwinian laboratory of multiple contact zones. Trends Ecol Evol 35, 1021-1036 (2020).

35. T. Giesecke, KD. Bennett, The Holocene spread of Picea Abies (L.) Karst. in Fennoscandia and adjacent areas. Journal of Biogeography 31(9), 1523-48 (2004).

36. H.A. Binney et al., Vegetation of Eurasia from the Last Glacial Maximum to present: Key biogeographic patterns. Quaternary Science Reviews 157, 80-97 (2017).

37. H.A. Binney et al., The Distribution of Late-Quaternary woody taxa in Northern Eurasia: Evidence from a new macrofossil database. Quaternary Science Reviews 28(23-24), 2445-64 (2009). 
38. D. Lehsten et al., Modelling the Holocene migrational dynamics of Fagus Sylvatica L. and Picea Abies (L.) H. Karst. Global Ecology and Biogeography 23(6), 658-68 (2014).

39. M. Latałowa, W.O. van der Knaap, Late Quaternary expansion of Norway spruce Picea abies (L.) Karst. in Europe according to pollen data. Quaternary Science Reviews 25(2122), 2780-2805 (2006).

40. G. Eriksson, I. Ekberg, An introduction to forest genetics. Swedish University of Agricultural Sciences (2001).

41. T. Günther et al., Population genomics of Mesolithic Scandinavia: Investigating early postglacial migration routes and high-latitude adaptation. PLoS Biology 16(1), e2003703 (2018).

42. B.M. Peter, D. Petkova, J. Novembre, Genetic landscapes reveal how human genetic diversity aligns with geography. Molecular Biology and Evolution 37(4), 943-51 (2020).

43. G. Hewitt, The genetic legacy of the Quaternary Ice Ages. Nature 405(6789), 907-13 (2000)

44. D. De Carvalho et al., Admixture facilitates adaptation from standing variation in the European aspen (Populus Tremula L.), a widespread forest tree. Molecular Ecology 19(8) 1638-50 (2010).

45. S.C. Bray et al., Ancient DNA identifies post-glacial recolonisation, not recent bottlenecks, as the primary driver of contemporary MtDNA phylogeography and diversity In Scandinavian brown bears. Diversity and Distributions 19(3), 245-56 (2013).

46. M. Jaarola, H. Tegelström, K. Fredga, Colonization history in Fennoscandian rodents. Biological Journal of the Linnean Society 68(1-2), 113-27 (1999).

47. J. Wang et al., A major locus controls local adaptation and adaptive life history variation in a perennial plant. Genome Biology 19(1), 1-17 (2018).

48. P. Androsiuk et al., Genetic status of Norway spruce (Picea abies) breeding populations for Northern Sweden. Silvae Genetica 63(2), 127 (2013).

49. M.A. Polezhaeva, M. Lascoux, V.L. Semerikov, Cytoplasmic DNA variation and biogeography of Larix Mill. in Northeast Asia. Molecular Ecology 19(6), 1239-52 (2010).

50. L. Schulte et al., Hybridization capture of larch (Larix Mill.) chloroplast genomes from sedimentary ancient DNA reveals past changes of Siberian forest. Molecular Ecology 21(3), 801-15 (2021).

51. V.L. Semerikov et al., Southern montane populations did not contribute to the recolonization of West Siberian Plain by Siberian larch (Larix sibirica): A range-wide analysis of cytoplasmic markers. Molecular Ecology 22(19), 4958-71 (2013).

52. N.V. Semerikov et al., Cytoplasmic DNA variation does not support a recent contribution of Pinus sylvestris L. from the Caucasus to the main range. Tree Genetics \& Genomes 16(4), 1-11 (2020). 
53. T. Giesecke, Holocene dynamics of the Southern boreal forest in Sweden. The Holocene 15(6), 858-72 (2005).

54. L. Kullman, Norway Spruce present in the Scandes Mountains, Sweden at 8000 BP: New light on Holocene tree spread. Global Ecology and Biogeography Letters 5(2), 94-101 (1996).

55. U. Herzschuh, Legacy of the Last glacial on the present-day distribution of deciduous versus evergreen boreal forests. Global Ecology and Biogeography 29(2), 198-206 (2020).

56. M. Hannerz, Genetic and seasonal variation in hardiness and growth rhythm in boreal and temperate conifers-a review and annotated bibliography. Report. Skogforsk (1998).

57. D. Grattapaglia, et al., Quantitative genetics and genomics converge to accelerate forest tree breeding. Frontiers in plant science 9, 1693 (2018).

58. A. Vidalis et al., Design and evaluation of a large sequence-capture probe set and associated SNPs for diploid and haploid samples of Norway spruce (Picea abies). bioRxiv [Preprint] (2018). https://www.biorxiv.org/content/10.1101/291716v1 (accessed 1 November 2021).

59. H. Li, R. Durbin, Fast and accurate long-read alignment with Burrows-Wheeler Transform. Bioinformatics 26(5), 589-95 (2010).

60. C.C. Chang et al., Second-Generation PLINK: Rising to the challenge of larger and richer datasets. GigaScience 4(1), 7 (2015).

61. K.J. Galinsky et al., Fast Principal-Component Analysis reveals convergent evolution of ADH1B in Europe and East Asia. The American Journal of Human Genetics 98(3), 456$72(2016)$.

62. R.R. Hudson, M. Slatkin, W.P. Maddison, Estimation of levels of gene flow from DNA sequence data. Genetics 132(2), 583-89 (1992).

63. G. Coop, D. Witonsky, A. Di Rienzo, J.K. Pritchard, Using environmental correlations to identify loci underlying local adaptation. Genetics 185(4), 1411-23 (2010).

64. F. Privé, K. Luu, B.J. Vilhjálmsson, M.G. Blum, Performing highly efficient genome scans for local adaptation with R Package Pcadapt Version 4. Molecular Biology and Evolution 37(7), 2153-54 (2020).

65. A. Alexa, J. Rahenfuhrer, TopGO: Enrichment analysis for gene ontology. Bioconductor Improv, 27, 1-26 (2009).

66. M. Tiret, P. Milesi, Statistical Peak Detection for GWAS. Zenodo. https://doi.org/10.5281/zenodo.5079556. Deposited 7 July 2021. 
A]

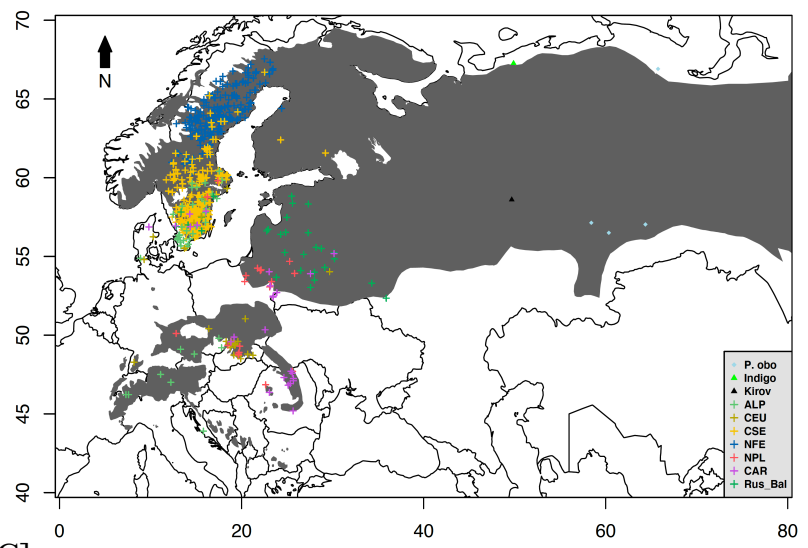

C]

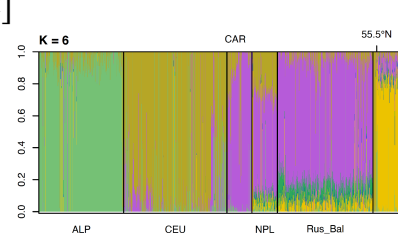

B]

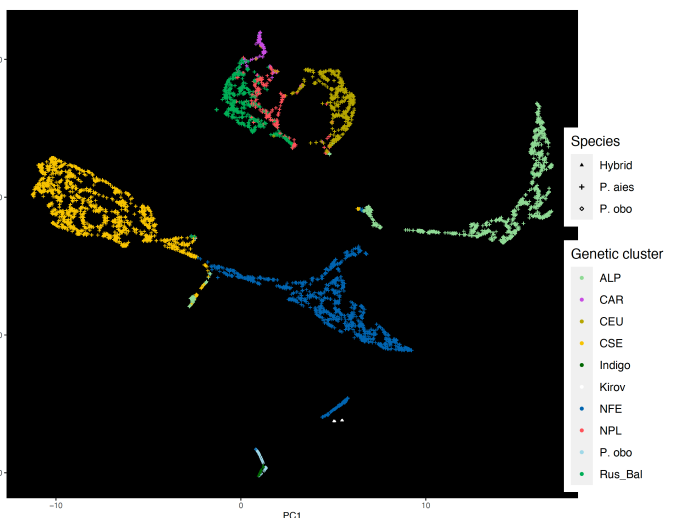

Figure 1: Population genetic structure of $\boldsymbol{P}$. abies. A) Sampling location of $P$. abies (plus signs: light green, Alpine (ALP); brown, Central Europe (CEU); purple Carpathian (CAR); red, northern Poland (NPL); dark green Russia-Baltics (Rus_Bal); yellow, southern Fennoscandia (CSE) and dark blue, northern Fennoscandia (NFE), $P$. obovata (diamonds, light blue) and hybrids (Indigo and Kirov) (black triangles). The shaded area corresponds to the distribution range of $P$.abies and $P$. obovata B) UMAP bi-dimensional plots, colors are the same as for panel A. C) Admixture plot for $K=6$. Samples from a same geographic origin were grouped. Swedish samples were ordered by latitude. Colors represent different 
A]

854

855

856

857

858

859

860

861

862

863

864

865

866

867
B]

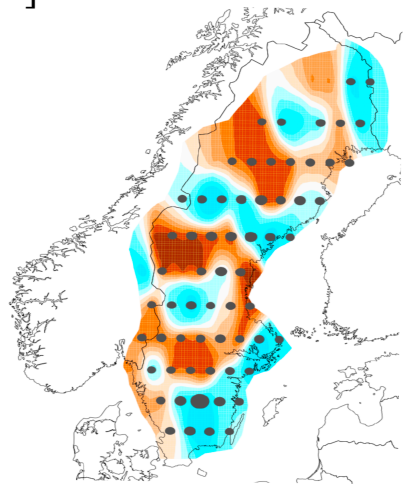

C]

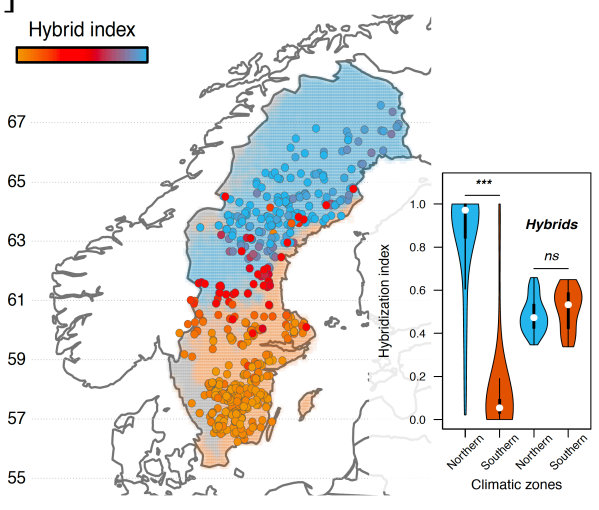

Figure 2: Fine genetic structure of the contact zone and relation to climate zones. A) Admixture proportions based on the best spatial model using conStruct $(\mathrm{K}=3)$. Colors represent different ancestry components. Close-by samples were grouped into "populations". B) Estimated effective migration surfaces (EEMS). Blue and brown areas respectively indicate regions with a higher or a lower effective migration rate than expected under a model of isolation by distance (IBD). Gray dots represent individual aggregations. C) The genetic contact zone overlaps with the transition between the two main climatic zones, the southern one (orange background) and the northern one (blue background). Dots represent tree locations and the color scale corresponds to the hybridization level (from 0 , full CSE, orange, to 1 , full NFE, blue). Violin plots represent the distribution of hybrid index within each of the two main climatic zones (all samples or only samples with $0.33>$ hybrid index $\left.<0.66 ;{ }^{\text {ns }}, p>0.05 ;{ }^{* * *}, p<0.001\right)$. 
A]

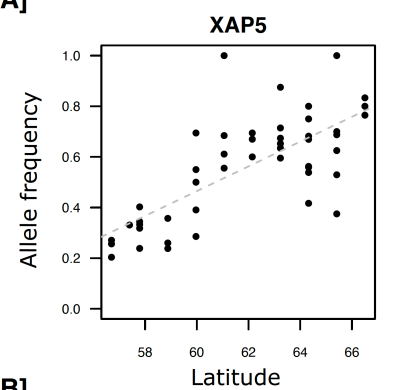

B]
Gigantea

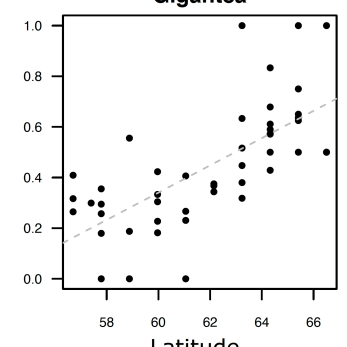

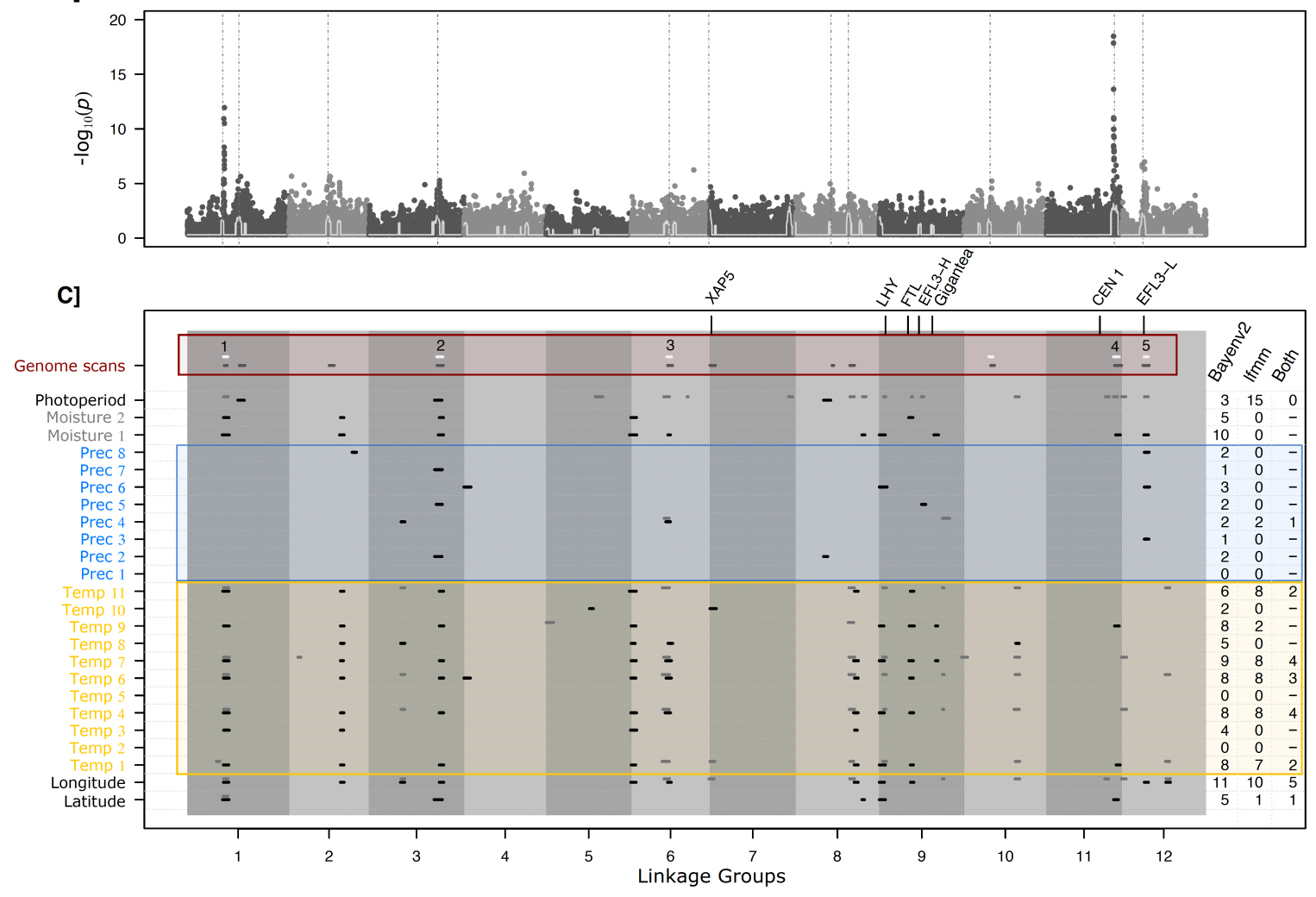

SSR1

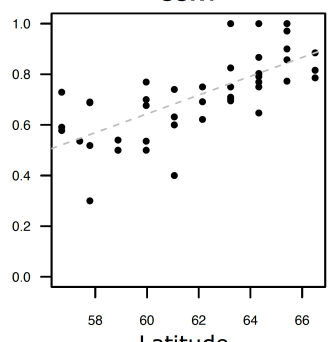

Latitude

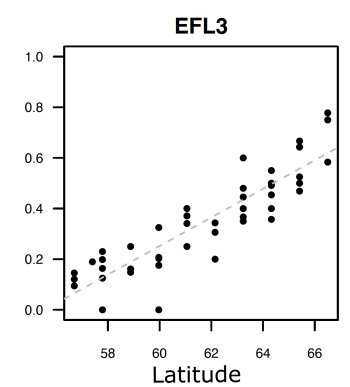

Latitude

Figure 3: Genome scans for differentiation outliers and genotype-environment association (GEA). A) Examples of allele frequency variation as a function of latitude for four candidate genes involved in the control of the circadian clock. B) Manhattan plot ($\log 10 p$-values) of genome scan for excess of differentiation (pcAdapt). Dark and light grey backgrounds delineate linkage groups. Vertical dotted grey lines represent regions enriched for low p-values, "peaks" in the profile. A detailed analysis is provided for each of the genome scan and GEA in Supplementary material 3. C) For each genome scan (white, $X^{T} X$; grey, pcAdapt) and genotype-environment-association (black, Bayenv2; dark grey, Ifmm2) significant peaks are localized on the Norway spruce genetic map. For each geographic and bioclimatic variable, the number of significant peaks is indicated on the right as well as the number of shared peaks. Numbers at the top of the graph identify significant peaks detected by the two genome scans methods and at least one GEA method. When possible, genes involved in the control of circadian clock were placed onto the genetic map. 


\begin{tabular}{lllcc}
\hline $\begin{array}{l}\text { Distance to } \\
\text { contact zone }\end{array}$ & AM $v s$ SI & IM $v s$ SC & M-homo $v s$ M-hetero & $\mathbf{N}_{\mathbf{e}}$-homo $v s \mathbf{N}_{\mathbf{e}}$-hetero \\
\hline close & $\operatorname{AM}(p=1.00)$ & $\operatorname{IM}(p=0.52)$ & M-homo $(p=0.89)$ & N-hetero $(p=0.88)$ \\
intermediate & $\operatorname{AM}(p=1.00)$ & $\operatorname{IM}(p=0.49)$ & M-homo $(p=0.95)$ & N-hetero $(p=0.93)$ \\
far & $\operatorname{AM}(p=1.00)$ & $\operatorname{IM}(p=0.55)$ & M-homo $(p=0.89)$ & N-hetero $(p=0.71)$ \\
\hline
\end{tabular}

Table 1: Pairwise comparison of different models with DILS for individuals at varying distance from the center of the contact zone. Demographic models: Strict Isolation (SI), 888 Ancient Migration (AM), Isolation with Migration (IM), Secondary Contact (SC), Homogeneous and Heterogeneous migration ( $\mathrm{Nm}$ ) (M-homo and M-hetero), and Homogeneous

890 and Heterogeneous effective population size $\left(\mathrm{N}_{\mathrm{e}}\right)\left(\mathrm{N}_{\mathrm{e}}\right.$-homo and $\mathrm{N}_{\mathrm{e}}$-hetero). The value 891 within parentheses, $p$, is the posterior probability of the best demographic model. Dis892 tance to contact zone is defined according to the hybrid index.

893 


\section{Online methods}

895

896 Sample collection: The study was based on 4769 spruce trees, originating mainly

897 from the Swedish Norway spruce ( $P$. abies) breeding base population (Figure 1).

898 Among them, 162 individuals were collected in natural populations across the Norway spruce natural distribution range (40 individuals from the hybrid zone between $P$. abies and $P$. obovata, 69 pure $P$. abies and 53 pure $P$. obovata) (8). The remaining trees (4607) were "plus" trees (trees of outstanding phenotype) sampled in Skogforsk (The Forestry Research Institute of Sweden) plantations across Sweden. A total of 873 of those trees lacked geographic information on their origin (Table S1). All trees were genotyped ((8), BioProject PRJNA511374 and (21), BioProject PRJNA731384) using an exome capture target re-sequencing strategy (40,018 diploid probes with 20bp-length were designed to capture 26,219 P. abies genes, (58)).

Single Nucleotide Polymorphism calling: Raw reads were mapped to the $P$. abies genome reference v1.0 (32) using BWA-MEN algorithm with default parameters (67). PCR duplicates were removed using SAMTOOLS v.1.2 (68) and Picard v1.141 (http://broadinstitute.github.io/picard), and INDELs were realigned using GATK v3-5.0 (62). Individual variants were identified using HaplotypeCaller v.3.6 (69) and individual g.vcf were merged using CombineGVCFs v.3.5.0. Variant quality score recalibration (VQSR) was applied following the same procedure as in (70). We additionally filtered SNPs according to four criteria. SNPs were removed if at least one of the following conditions was met: (i) Root Mean Square of the mapping quality $(\mathrm{MQ})<40$; (ii) Allele depth $(\mathrm{AD})<2$; (iii) genotyping coverage across individuals $<20 \%$ and (iv) the site has more than two alleles. Individuals with more than $50 \%$ missing data were also removed $(\mathrm{N}=282)$.

The filtered dataset included 4508 individuals and 504,110 SNPs. Those SNPs were annotated based on the most recent genome annotation available for P. abies (v1.0, http://congenie.org/): 205,337 (41\%) SNPs are within introns, $108,300(21 \%)$ are in intergenic regions and 190,463 are in the exons $(38 \%)$ of which $63 \%$ are synonymous variants (24\% of total SNPs) and $37 \%$ are nonsynonymous variants ( $14 \%$ of total SNPs). 
926 Population structure and genotype assignment: For population structure anal-

927 yses, sites in high linkage disequilibrium $\left(r^{2}>0.2\right)$ as well as singletons were re-

928 moved using PLINK v.1.9 (60). Among the remaining SNPs, 155,211 putatively

929 neutral SNPs (i.e. within introns and intergenic regions) were kept for demographic

930 analyses.

931 Principal component analysis. We first conducted a principal component analysis

932 (PCA) using EIGENSOFT with default parameters (v.7.2.0,

933 https://github.com/DreichLab/EIG, (61). We then subset the dataset to insure even

934 number of individuals $(\mathrm{N}=80)$ in the different genetic clusters and re-ran the PCA.

935 The procedure was repeated eight times and a similar clustering was obtained in

936 all runs (SI Appendix, Figure S1).

937 Genotype assignment. The same Random Forest classification procedure as in (8)

938 was then used to infer the geographic origins of the 873 trees whose geographic

939 coordinates were unknown. The assignment was based on genotype similarity on

940 the first five principal components of the PCA ("Random Forest" classification

941 model in R version 3.5.3, 'randomForest' v.4.6-14 package (74,75). The $2572 P$.

942 abies trees with documented geographical origins and falling in the center of each

943 genetic cluster in the PCA were used as training set. The procedure was repeated

944200 times with 8000 iterations to estimate the accuracy of each assignment. If a

945 tree was assigned to the same genetic cluster more than $98 \%$ times, it was con-

946 sidered as belonging to that genetic cluster.

947 Model-based clustering. We first estimated population structure using the unsu-

948 pervised genetic clustering algorithms implemented in ADMIXTURE v1.3 (25) with

949 ten-fold cross validation and 200 bootstraps. The K-value with the lowest cross

950 validation error was retained as the "best" number of theoretical ancestral clusters,

951 but we also reported the results for K varying from 2 to "best-K"+1 as identifying

952 the "true" number of clusters remains an elusive problem $(3,76)$. As for PCA, the

953 whole analysis was repeated on subsets with even number of individuals randomly

954 sampled in each of $P$. abies genetic cluster. 
$955 F_{S T}$ estimates. Pairwise fixation indices (Hudson's estimator of $F_{\mathrm{ST}}$, (62)) were then

956 estimated between $P$. obovata, admixed $P$. abies $\times P$. obovata populations and the

$957 P$. abies genetic clusters defined through the PCA analysis.

959 For the following analyses, only trees that were of confirmed Swedish origin (base

960 on genetic clustering) and with known geographic coordinates were considered ( $N$

$961=1758)$. We filtered the SNPs dataset to remove loci with $>50 \%$ missing geno-

962 types, newly-generated invariant sites and singletons. We retained 113,748 un-

963 linked putatively neutral SNPs (from introns and intergenic regions).

965 Spatialized analyses of genetic variation of Norway spruce across Sweden

966 The Swedish $P$. abies populations are, by and large, continuously distributed and

967 there is a high level of long-distance gene flow (77). To consider both discrete

968 clusters and continuous distribution of the genetic variation of Norway spruce

969 across Sweden, we used the conStruct software v. 1.03 (3) that combines model-

970 based clustering algorithms with an isolation by distance model. The 1758 geo-

971 referenced samples with genetic Swedish origin were assigned to 47 populations.

972 These "artificial populations" were defined by grouping trees from close geographic

973 origins $(\mathrm{N}>5)$. We ran conStruct using $\mathrm{K}=1$ to $\mathrm{K}=7$ for one chain and each with

974 100,000 Markov chain Monte Carlo (MCMC) iterations, and compared spatial and

975 nonspatial models using cross validation across 10 replicates.

976 Isolation-by-distance and identification of barriers to gene flow:

977 Estimating effective migration surfaces. To identify corridors or barriers to gene 978 flow, we used EEMS software (v. 0.0 .9000 (26)). It estimates effective migration

979 surfaces (EEMS) from geographically indexed samples. Sample coordinates and 980 pairwise genetic dissimilarity were used to identify regions with faster or slower 981 change in genetic similarities than predicted under an isolation by distance model.

982 The overall Norway spruce habitat in Sweden was divided into triangular grids cor983 responding to different deme numbers (30,50, 80 and 100). Each sample was 984 assigned to the closest point on the grid. To test the stability of the results the 985 program was run five times with 10,000,000 MCMC iterations and 5,000,000 burn- 
986 ins for each deme size. Following authors guideline, we combined estimates over

987 different grids.

988

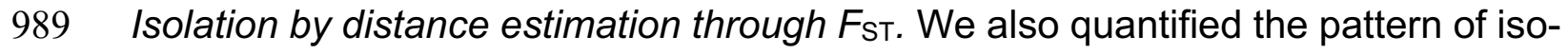

990 lation by distance by regressing a function of $F_{\mathrm{ST}}(27)$ over the logarithm of the

991 distance between pairs of populations (we used the same populations as those

992 defined for the conStruct analysis):

993

$$
\frac{F \hat{S} T_{l}}{\left(1-F_{S} T_{l}\right)}=\beta \cdot \ln \left(x_{i}\right)+\alpha+\varepsilon_{i}[\text { eq. } 1]
$$

994

995 For each pair of sub-populations $i, F_{S T_{l}}^{\wedge}$ was estimated using vcftools (v0.1.13, (74)),

$996 x_{i}$ is the geodesic distance separating a pair of populations (in km, "geosphere" $R$

997 package v1.5-10, (81)), $\beta$ is the slope of the regression, $\alpha$ is the intercept and $\varepsilon_{i}$ is

998 the error term. According to (27), independently of the scale, the slope is inversely

999 proportional to the product of population density $D$, by the second moment of dis-

1000 persal distance $\sigma^{2}: \beta=1 /\left(4 \pi D \sigma^{2}\right)$. In Sweden Norway spruce distribution is al-

1001 most continuous and an even density can be assumed. The "slope" estimate

$10024 \pi D \sigma^{2}$ can thus be interpreted as a neighborhood size, individuals within a neigh-

1003 borhood mating randomly.

\section{The contribution of linked selection to the contact zone.}

1007 In order to test whether linked selection contributed to the establishment and 1008 maintenance of the contact zone, we used the program DILS (28). Briefly, DILS 1009 implements single-population and two-population demographic models and identi1010 fies the most likely demographic scenario with and without linked selection. In the 1011 case of two-population models that diverged T generations back, four demographic 1012 models are possible, namely Strict Isolation (SI), Ancient Migration (AM), Isolation 1013 with Migration (IM) and Secondary Contact (SC). The effect of linked selection is 1014 estimated through its effect on the distribution of the effective population size, $\mathrm{N}_{\mathrm{e}}$, 1015 or the effective migration rate, $\mathrm{Nm}$, along the genome. DILS uses Approximate 
1016 Bayesian Computations (ABC) and computes summary statistics on the real da1017 taset (nucleotide diversity pi, Tajima's D, Watterson's theta, and summary statistics 1018 approximating the joint Site Frequency Spectrum), and compare them to a pre1019 simulated reference table made of 10,000 simulations of 1,000 loci for each demo1020 graphic scenario. Model comparison is then done with a random forest of 1,000 1021 trees. An error rate per decision tree (e) is estimated, and the posterior probability 1022 is computed as 1-e. The population growth was considered constant, mutation rate 1023 fixed to $2.763 .10^{-8}$, and priors with a log-Uniform distribution for effective popula1024 tion size (from 100 to 500,000), time of split (from 100 to 1,750,000 generations), 1025 and effective migration rate (from 0.4 to 40 ). Considering that distance to the con1026 tact zone might influence demographic inferences (e.g., hybrids have different his1027 tory than pure individuals), we created three different datasets of 20 individuals (10 1028 from NFE and 10 from CSE) as inputs for DILS depending on the distance to the 1029 contact zone. Distance to the contact zone was expressed in terms of hybrid index, 1030 the center of the contact zone being arbitrarily defined as the location where the 1031 hybrid index is 0.5 . The dataset "far" comprised individuals with a hybrid index 1032 lower than 0.04 or higher than 0.96; the dataset "intermediate" comprised individ1033 uals with a hybrid index between 0.14 and 0.36 or between 0.64 and 0.87 ; finally, 1034 the dataset "close" comprised individuals with a hybrid index between 0.37 and 1035 0.63. Individuals of each dataset were randomly sampled from the respective 1036 range of hybrid index. Following software guidelines, a subset of coding regions 1037 was used: 1708 coding regions, corresponding to $1 \%$ of the exome (from 500 ran1038 domly sampled scaffolds).

1040 Abiotic environment characterization and climatic zones definition: To as1041 sess whether the contact zone between the main genetic clusters corresponded to 1042 a shift in abiotic conditions across Sweden, we defined climatic zones based on 104319 bioclimatic records (Chelsa database v1.2, http://chelsa-climate.org/, 30 arc1044 second resolution) using an unsupervised clustering approach (i.e. without any a 1045 priori). Values for each bioclimatic variable were extracted for each geographic 1046 coordinate corresponding to land in Sweden and to a grid with nodes every 0.1 
1047 degree of latitude and longitude. A principal component analysis was carried out 1048 on these data ("PCA" function, R package "FactoMineR" v1.42, (83)) followed by 1049 a hierarchical ascendant clustering approach ("HCPC" function, R package "Fac1050 toMineR"). The optimal number of clusters (Qopt) was defined based on the inertia $1051(I)$ growth between increasing number of clusters $(q)$ :

$$
Q_{o p t}=\min \left(\frac{I_{q-1}-I_{q}}{I_{q}-I_{q+1}}\right) \text { eq.2 }
$$

\section{Testing for local adaptation}

1054 Different approaches were used to test for the presence of local adaptation at the 1055 genomic level and to detect association between genomic polymorphisms and en1056 vironmental variables. In line with our analysis of population structure we used two 1057 types of approaches. The first family of methods assumes the presence of popu1058 lations $\left(n_{p o p}=47\right)$ and uses the pattern of allele frequencies within and between 1059 these populations to make inference on natural selection or identify genomic areas 1060 associated to environmental variation. In contrast, methods from the second group 1061 are based on individuals and do not require the a priori clustering of individuals 1062 into a finite number of populations. The same genomic dataset was used for all 1063 subsequent analyses and all SNPs (including also coding sites), with a minimum 1064 allele frequency (MAF) higher than 0.05 and less than $50 \%$ missing data were kept $1065\left(n_{S N P}=142,766\right)$.

1066 Detecting excess of differentiation: We used the Bayenv2 software $(41,83)$ to 1067 compute the $X^{T} X$ statistic, an analogue to $F_{S T}$ based on standardized allele fre1068 quencies corrected for population structure. To compute the covariance matrix of 1069 allele frequencies across the 47 populations, 20 sets of 8,000 non-coding and un1070 linked loci were randomly selected from the same dataset as the one used in the 1071 conStruct analysis. The covariance matrix was obtained by averaging over the last 1072 matrices generated by the 20 independent runs of 100,000 MCMC iterations. Only 1073 SNPs with a $X^{T} X$ higher than $\overline{X^{T} X}+3^{*}$ SD where SD is the standard deviation of the $1074 X^{T} X$ distribution were considered significant (> 0,993 quantile).

1075 We also used a PCA-based outlier detection method implemented in the "pcAdapt" 1076 v4.3.2 R package $(30,84)$. The method assumes that the main part of the SNPs 
1077 variation along principal component axes reflects demographic processes and 1078 population structure. Extreme values correspond to outliers SNPs that are pre1079 sumed to be in the vicinity of SNPs involved in adaptation. To ensure that the ob1080 served pattern is not driven by a small region with extended linkage disequilibrium, 1081 PCA loadings were checked. To control for false positives, only SNPs with FDR $q$ 1082 value $<0.1$ were considered as putatively involved in local adaptation.

1083 Genotype-environment associations: In order to further characterize the genetic 1084 basis of local adaption in Norway spruce, we conducted genotype-environment 1085 associations (GEAs). As for the detection of excess of differentiation, we used two 1086 different approaches, one population-based, "Bayenv2" and one individual-based, 1087 "Ifmm2" (30). The same 19 Chelsa bioclimatic variables as those used to define 1088 the climatic zone were used for each tree location. Three additional climatic varia1089 bles were computed: (i) annual heat-moisture index (AHM, annual mean tempera1090 ture / (total annual precipitations/100)), (ii) summer heat-moisture index (SHM, av1091 erage temperatures of warmest month / (total precipitations of warmest quar1092 ter/100)) and (iii) average day length difference between June and January; the 1093 latter was used as a proxy for the growth period (SI Appendix Table S2).

1094 Bayenv2 tests for a correlation between allele frequencies and an environmental 1095 variable by using a Bayesian generalized linear mixed model. A variance-covari1096 ance matrix of allele frequencies is incorporated as random effect to correct for 1097 population structure. For each climatic variable plus latitude and longitude, both 1098 Bayes Factor (BF) and Spearman's rho correlation coefficient were computed to 1099 measure the intensity of the association between allele frequency variation and 1100 environmental variation. For each climatic variable, the following filtering (based 1101 on Bayes factor and Spearman's rho) was applied to retain only the most relevant 1102 SNPS: (a) the SNPs were ranked according to their Bayes factor (BF) and a SNP 1103 was retained if $\mathrm{i}$ ) its BF > 100 (very strong strength of evidence according to (85) 1104 or, if ii) its BF > 20 (strong strength of evidence) and it was within the $0.1 \%$ highest 1105 BF.

1106 Latent factor mixed models were also used to test for associations between the 1107 set of environmental / geographic variables and SNPs variation. The "Ifmm2" 
1108 function ("LEA" R package) was used to estimate latent factors based on an exact

1109 least-squares approach. Missing genotypes were imputed using the "impute" func-

1110 tion following author recommendations. The number of latent factors to be included

1111 in the analysis was determined using the "snmf" function (MCMC, 10 repetitions,

$11126 \%$ of dataset masked per repetitions). It was defined as being the one minimizing

1113 the cross-entropy criterion across all runs. The latent factors were used to correct

1114 for population structure in linear regressions between genotypes and environmen-

1115 tal variables; $p$-values were recalibrated by using genomic control after correction

1116 for confounding effect from population structure ("Ifmm2.test" function). To control

1117 for false positives, only SNPs with a $q$-value < 0.1 were considered ("qvalue"

1118 v2.20.0 R package, method "fdr", Storey et al. 2020).

1119 Candidate genes putative functions: Gene ontology (GO) enrichment was per-

1120 formed using the 'topGO' R package (v2.44.0; (86). Annotation from ConGenIE

1121 (the Conifer Genome Integrative Explorer, http://congenie.org/) was used as refer-

1122 ence (i.e. custom input). For various lists of candidate genes, defined through both

1123 genome-scans or GEA, enrichment of genes in particular GO terms for biological

1124 processes (BP) was assessed using Kolmogorov-Smirnov's tests "elimKS". rrvgo

1125 (87) was then used to summarize gene ontology terms by collapsing redundant

1126 terms across hierarchical levels using Arabidopsis thaliana as a reference for GO

1127 term (v.3.13.0, (88)).

1128 Genetic map positioning of loci putatively involved in local adaptation: About

$112960 \%$ of all SNPs were successfully positioned onto the $P$. abies consensus genetic

1130 map (33). We developed a new approach to identify regions enriched for outliers

1131 (either low p-values in pcaAdap, and Ifmm2 analyses or high Bayes factor for

1132 Bayenv2). The method (66) is described and freely accessible at

1133 https://github.com/mtiret/gwas-snp-detection. For each genome scan or GEA, we

1134 searched for regions with more outlier SNPs (top 5\% of the distribution) than ex-

1135 pected under a random distribution. In a first step, we generated a null expectation

1136 through randomization (10,000 runs) to determine the maximum number of outliers

1137 expected by chance within each 300 bp window. This number was then used as a

1138 threshold for peak detection in the actual data. Finally, to be conservative, only 
1139 peaks containing at least one SNP detected as significant after correction for mul-

1140 tiple testing were considered as candidate regions.

1141

1142

1143

1144

1145

1146

1147

1148

1149

1150

1151

1152

1153

1154

1155

1156

1157

1158

1159

1160

1161

1162

1163

1164

1165

1166

1167

1168

1169

1170

1171

1172

1173

1174

1175

1176

1177

1178

1179

1180

\section{References}

67. H. Li, R. Durbin, Fast and Accurate Long-Read Alignment with BurrowsWheeler Transform. Bioinformatics 26(5), 589-95 (2010).

68. H. Li et al., The Sequence Alignment/Map Format and SAMtools. Bioinformatics 25(16), 2078-79 (2009).

69. A. McKenna et al., The Genome Analysis Toolkit: A MapReduce Framework for Analyzing next-Generation DNA Sequencing Data. Genome Research 20(9), 1297-1303 (2010).

70. G.A. van der Auwera et al., From FastQ Data to High-Confidence Variant Calls: The Genome Analysis Toolkit Best Practices Pipeline. Current Protocols in Bioinformatics 43(1), 11-10 (2013).

71. J. Baison et al., Genome-Wide Association Study Identified Novel Candidate Loci Affecting Wood Formation in Norway Spruce. Plant Journal 100(1), 83-100 (2019).

72. C.C. Chang et al., Second-Generation PLINK: Rising to the Challenge of Larger and Richer Datasets. GigaScience 4(1), 7 (2015).

73. K.J. Galinsky et al., Fast Principal-Component Analysis Reveals Convergent Evolution of ADH1B in Europe and East Asia. The American Journal of Human Genetics 98(3), 456-72 (2016).

74. A. Liaw, M. Wiener, Classification and Regression by RandomForest. $R$ news 2(3), 18-22 (2002).

75. R Core Team, R: A Language and Environment for Statistical Computing. R Foundation for Statistical Computing, Vienna Austria (2013).

76. J.K. Pritchard, M. Stephens, P. Donnelly, Inference of Population Structure Using Multilocus Genotype Data. Genetics 155(2), 945-59 (2000). 
77. J. Burczyk, A. Lewandowski, W. Chalupka, Local Pollen Dispersal and Distant Gene Flow in Norway Spruce (Picea Abies [L.] Karst.). Forest Ecology and Management 197(1-3), 39-48 (2004).

78. D. Petkova, J. Novembre, M. Stephens, Visualizing Spatial Population Structure with Estimated Effective Migration Surfaces. Nature Genetics 48(1), 94-100 (2015).

79. B.S. Weir, C.C. Cockerham, Estimating F-Statistics for the Analysis of Population Structure. Evolution 38(6), 1358 (1984).

80.P. Danecek et al., The Variant Call Format and VCFtools. Bioinformatics 27(15), 2156-58 (2011).

81. R.J. Hijmans, E. Williams, C. Vennes, Geosphere: Spherical Trigonometry. $R$ package version 1.5-14. https://cran.r-project.org/package=geosphere (2021).

82. S. Lê, J. Josse, F. Husson, FactoMineR: An R Package for Multivariate Analysis. Journal of Statistical Software 25(1), 1-18 (2008).

83. G. Coop, D. Witonsky, A. Di Rienzo, J.K. Pritchard, Using Environmental Correlations to Identify Loci Underlying Local Adaptation. Genetics 185(4), 1411-23 (2010).

84.F. Privé, K. Luu, B.J. Vilhjálmsson, M.G. Blum, Performing Highly Efficient Genome Scans for Local Adaptation with R Package Pcadapt Version 4. Molecular Biology and Evolution 37(7), 2153-54 (2020).

85. R.E. Kass, A.E. Raftery, Bayes Factors. Journal of the American Statistical Association 90(430): 773-95 (1995).

86. A. Alexa, J. Rahenfuhrer, TopGO: Enrichment Analysis for Gene Ontology. Bioconductor Improv, 27, 1-26 (2009).

87. S. Sayols, rrvgo: A Bioconductor Package to Reduce and Visualize Gene Ontology Terms. R package version, https://ssayols.github.io/rrvgo (2020).

88. M. Carlson, Org.At.Tair.Db: Genome Wide Annotation for Arabidopsis. R package version 3.7 .0 (2018). 